\title{
Tuberculosis y su Relación con los Espacios Periurbanos de Lima - Perú: análisis demográfico y socio-ambiental
}

\author{
Tuberculosis and its Relationship with the Periurban Spaces of Lima - Perú: \\ demographic and socio-environmental analysis
}

\section{Tuberculose e a sua Relação com os Espaços Periurbanos de Lima - Perú: análise demográfica e socioambiental}

\author{
Jhomelin Milagros Flores Bordais ${ }^{1}$ \\ Exzolvildres Queiroz Neto \\ Carmen Justina Gamarra ${ }^{3}$
}

\begin{abstract}
RESÚMEN: La tuberculosis es una enfermedad que viene causando gran impacto en las diversas sociedades del mundo, principalmente en aquellas en situación de vulnerabilidad. Esta enfermedad es causada por el Mycobacterium tuberculosis o comúnmente llamado bacilo de Koch, en honor a su descubridor. La tuberculosis en el Perú concentra el mayor número de casos en los diversos distritos ubicados, prioritariamente, en la periferia limeña. Este estudio objetivó analizar la relación que existe entre la tuberculosis y su presencia en los espacios periurbanos de Lima - Perú, espacios que fueron formados principalmente a partir de la migración y los conflictos internos suscitados en el país. Se destaca los aspectos demográficos y socio-ambientales del espacio territorial en donde situaremos a la tuberculosis como enfermedad predominante. Se determina las características de las condiciones de vida de sus habitantes por medio de los aportes de la geografía de la salud que incluye la conceptualización de la vivienda, la pobreza, la inequidad social y la configuración de la exclusión social. Finalmente, este estudio nos permitió afirmar la relación directa de la tuberculosis con el espacio en el que se desarrolla, ambientes precarios que contribuyen a la diseminación de la enfermedad.
\end{abstract}

PALABRAS CLAVES: Mycobacterium tuberculosis. Geografía médica. Salud pública.

ABSTRACT: Tuberculosis is a disease that has been causing great impact in the various societies of the world, mainly in those in a situation of vulnerability. This disease is caused by Mycobacterium tuberculosis or commonly called Koch Bacillus, in honor of its discoverer. Tuberculosis in Peru concentrates the largest number of cases in the various districts located, primarily, in the periphery of Lima. This study objectively analysed the relationship between tuberculosis and its presence in the peri-urban areas of Lima - Peru, spaces that were formed mainly from migration and internal conflicts raised in the country. It highlights the demographic and socio-environmental aspects of territorial space where we will place tuberculosis as a predominant disease. It determines the characteristics of

\footnotetext{
${ }^{1}$ Universidad Federal de Integración Latinoamericana. Rua Belo Horizonte, 54 - Vila C - Foz do Iguaçu/PR. jhomelinflores@gmail.com.

${ }^{2}$ Universidade Federal da Integração Latino-Americana. Av. Silvio Américo Sasdelli, 1842 - Vila A, Foz do Iguaçu - PR, 85866-000. eqngeo@gmail.com.

${ }^{3}$ Universidade Federal da Integração Latino-Americana. Av. Silvio Américo Sasdelli, 1842 - Vila A, Foz do Iguaçu - PR, 85866-000. carmen.gamarra@unila.edu.br.
} 
the living conditions of its inhabitants through the contributions of the geography of health that includes the conceptualization of housing, poverty, social inequity and the configuration of social exclusion. Finally, this study allowed us to affirm the direct relationship of tuberculosis with the space in which it develops, precarious environments that contribute to the spread of the disease.

KEYWORDS: Mycobacterium tuberculosis. Geography medical. Public health.

RESUMO: A tuberculose é uma doença que está causando grande impacto nas diferentes sociedades do mundo, principalmente naquelas em situação de vulnerabilidade. Esta doença é causada pelo Mycobacterium tuberculosis ou comumente chamado bacilo de Koch, em homenagem ao seu descobridor. A tuberculose no Peru concentra o maior número de casos nos vários distritos localizados, principalmente, na periferia de Lima. Este estudo teve como objetivo analisar a relação entre a tuberculose e sua presença nos espaços periurbanos de Lima - Peru, espaços que foram formados principalmente pela migração e os conflitos internos no país. Destacam-se os aspectos demográficos e socioambientais do espaço territorial onde colocaremos a tuberculose como doença predominante. Determinam-se as características das condições de vida de seus habitantes através das contribuições da geografia da saúde que inclui a conceitualização da habitação, da pobreza, da desigualdade social e a configuração da exclusão social. Finalmente, este estudo nos permitiu afirmar a relação direta da tuberculose com o espaço em que se desenvolve, ambientes precários que contribuem para a disseminação da doença.

PALAVRAS-CHAVE: Mycobacterium tuberculosis. Geografía médica. Saúde pública.

\section{INTRODUCCIÓN}

El análisis demográfico y socio-ambiental de los espacios periurbanos de Lima - Perú y su relación con la tuberculosis es hoy en día un reto importante para las diversas disciplinas con miras a entender su compleja formación y como se relacionan con la ocurrencia de la enfermedad. La rápida y extraordinaria expansión de estos espacios, que ocurrió no solo en el Perú sino también en toda América Latina, nos lleva a un profundo análisis de los fenómenos sociales que vinieron con dicha expansión que nació principalmente con las migraciones del campo a la ciudad, proceso que acontecía en el interior del país en paralelo con los grandes movimientos migratorios que ocurrían en toda América Latina.

En 1945, a finales de la segunda guerra mundial, se despertó la idea de que todos los países debían vivir de forma centralizada para combatir la pobreza debido a la creciente población, cabe mencionar que por esa época la pobreza se tornaba un problema de carácter mundial, las personas empezaron a dejar sus ciudades para migrar de las zonas rurales hacia los principales centros de los países. En el caso del Perú el proceso migratorio se fue dando de manera progresiva, el despojo de tierras y ausencia del Estado en los lugares alejados de las ciudades obligaron a muchas personas a dejar sus lugares de residencia para poblar otros con mayores expectativas de vida (MATOS, 1986). 
La migración aceleró el proceso de urbanización en la ciudad de Lima, la gran cantidad de personas que provenían del interior del país desbordaron Lima, expandiéndose continuamente hasta lograr la cercanía y ocupación de los espacios rurales, siendo primero la ocupación de las áreas libres hasta llegar a poblar los cerros circundantes de la ciudad, llegando a formar lo que hoy en día es: Lima Este, Lima Norte y Lima Sur (MATOS, 2004).

La Organización Mundial de la Salud (ORGANIZACIÓN MUNDIAL DE LA SALUD, 2016), indica que la tuberculosis es una enfermedad que se concentra principalmente en zonas en situación de pobreza y extrema pobreza, características de los espacios periurbanos. En estos espacios en particular, la baja calidad de vida y el hacinamiento tienen el poder de incrementar la diseminación de la bacteria y la exposición de las personas susceptibles a los casos infecciosos de la enfermedad. El informe de la Organización Panamericana de la Salud (ORGANIZACIÓN PANAMERICANA DE SALUD, 2012), respecto al número de casos nuevos de tuberculosis en la región de las Américas, indica que Brasil ocupa el primer lugar con un registro de 83000 casos nuevos al año, seguido por Perú que presenta 30000 casos nuevos al año, de los cuales el $60 \%$ de casos se concentran en Lima y Callao (PERÚ, 2014).

Por todo lo expuesto, encontramos elementos de estudio que nos permiten relacionar el proceso migratorio, la formación de zonas periurbanas y la ocurrencia de la tuberculosis en Lima - Perú. Podemos decir entonces que abordar el tema de la salud y la enfermedad desde la óptica de la geografía de la salud será importante para explicar el comportamiento social de la tuberculosis y entender de forma más amplia su ocurrencia en determinados espacios y situaciones de modos de vida. Entender lo propuesto será fundamental para orientar las intervenciones de lucha contra la tuberculosis en los espacios periurbanos de Lima.

Por ello, el presente trabajo fue organizado en tres partes; inicialmente serán abordados los aspectos de territorio y suelo, esta parte estará dividida en territorio, superficie territorial y densidad poblacional y uso y ocupación del suelo, donde se hará la descripción y análisis de cada uno de ellos; en la segunda parte se abordará el proceso migratorio y la formación de zonas periurbanas, dividiéndolos en los siguientes temas: migración y aspectos históricos, urbanización y espacio periurbano, en esta parte se caracterizará el espacio a estudiar; y para finalizar, en la tercera parte, abordaremos la geografía de la salud enfocando la tuberculosis, donde trataremos las relaciones del espacio geográfico (espacio periurbano) con la ocurrencia de la enfermedad, dividiéndolo en los siguientes temas: ocupación del suelo y vivienda, condiciones de vida: pobreza e inequidad, necesidades básicas y exclusión social. 


\section{TERRITORIO Y SUELO}

\section{Territorio}

El término territorio según la Constitución Política del Perú (PERÚ, 1993) es el espacio geográfico donde interactúa un grupo social y se considera un elemento importante para el desarrollo de la vida; es el soporte físico de la Nación y del Estado. En dicho espacio geográfico el Estado ejerce sus potestades y está constituido por el suelo, subsuelo, espacio aéreo y dominio marítimo que comprende el mar adyacente a sus costas, así como su lecho y subsuelo hasta la distancia de las 200 millas marinas medidas desde las líneas de base que establece la ley. El territorio peruano se torna altamente heterogéneo, presentando tres regiones naturales: Costa (Chala), Sierra (Andina) y Selva (Amazonía), con una alta correspondencia biogeográfica relacionados con el clima y la biodiversidad. El Instituto Nacional de Estadística e Informática (2013) indica que los problemas territoriales en estas regiones por ocupación y uso del suelo generan deterioro de los ecosistemas, desarrollo desigual, falta de competitividad, exclusión territorial, exclusión social, pobreza, vulnerabilidad y amenaza natural y antrópica.

Lima Metropolitana pertenece a la región costera del Perú, es la capital política del país donde se ubica el primer puerto y aeropuerto, consolidándose como el principal centro económico del país y reforzando su centralidad por poseer una ubicación privilegiada de centro geográfico en la costa peruana, poseyendo fronteras equidistantes de norte a sur, así también por su ubicación estratégica en la cuenca del Pacifico que favorece sus relaciones externas. Lima Metropolitana está "[...] conformada por la gran conurbación integrada por los centros urbanos de las provincias de Lima y Callao [...]" (POBLACIÓN..., 2017).

La provincia de Lima es hoy en día una gran metrópoli que integra a 43 distritos distribuidos en cuatro grandes zonas: Lima Centro, Lima Este, Lima Norte y Lima Sur (antes denominadas "conos"). Lima se torna una ciudad heterogénea por su configuración territorial, que abarca zonas urbanas, periurbanas y rurales dentro de su espacio consolidado, caracterizándose por ser extensa, segregada y desigual, que aún se encuentra en proceso de cambio de uso del suelo ocupado y de sus dinámicas económicas y sociales.

\section{Superficie territorial y densidad poblacional}

La superficie del territorio nacional peruano es de 1 millón 285 mil 215,60 kilómetros cuadrados, ubicándose en uno de los 19 países más extensos del mundo. La Selva es la región más extensa de nuestro país, ocupa el 60,3\% del territorio peruano y alberga al $14,0 \%$ de la población total, está conformada por dos zonas definidas: la Selva Alta o Ceja 
de Montaña, y el llano amazónico o Selva Baja. La Costa es la región más densamente poblada, tiene $11,7 \%$ del territorio y contiene al $56,3 \%$ de la población. La Sierra cubre el $27,9 \%$ del territorio nacional incluyendo al $29,7 \%$ de la población. La superficie territorial de Lima es de 34 mil 828,12 kilómetros cuadrados, ocupando el 2,7\% del territorio nacional (INSTITUTO NACIONAL DE..., 2015).

Al 30 de junio del 2015 el INEI estimó la población peruana en 31 millones 151 mil 643 habitantes, de los cuales 15 millones 605 mil 814 son hombres y 15 millones 545 mil 829 son mujeres. Lima es el departamento con mayor cantidad de habitantes, presentando 9 millones 835 mil habitantes, lo que representa el 32\% de la población del país. Dentro del departamento de Lima, la provincia de Lima (con el mismo nombre) alberga el mayor número de habitantes, distribuidos en las zonas de Lima Centro, Lima Este, Lima Norte y Lima Sur, presentando 8 millones 890 mil 792 habitantes; seguida por la provincia constitucional del Callao con 1 millón 13 mil 935 habitantes (figura 1).

Figura 1 - Mapa Territorial de Lima Metropolitana

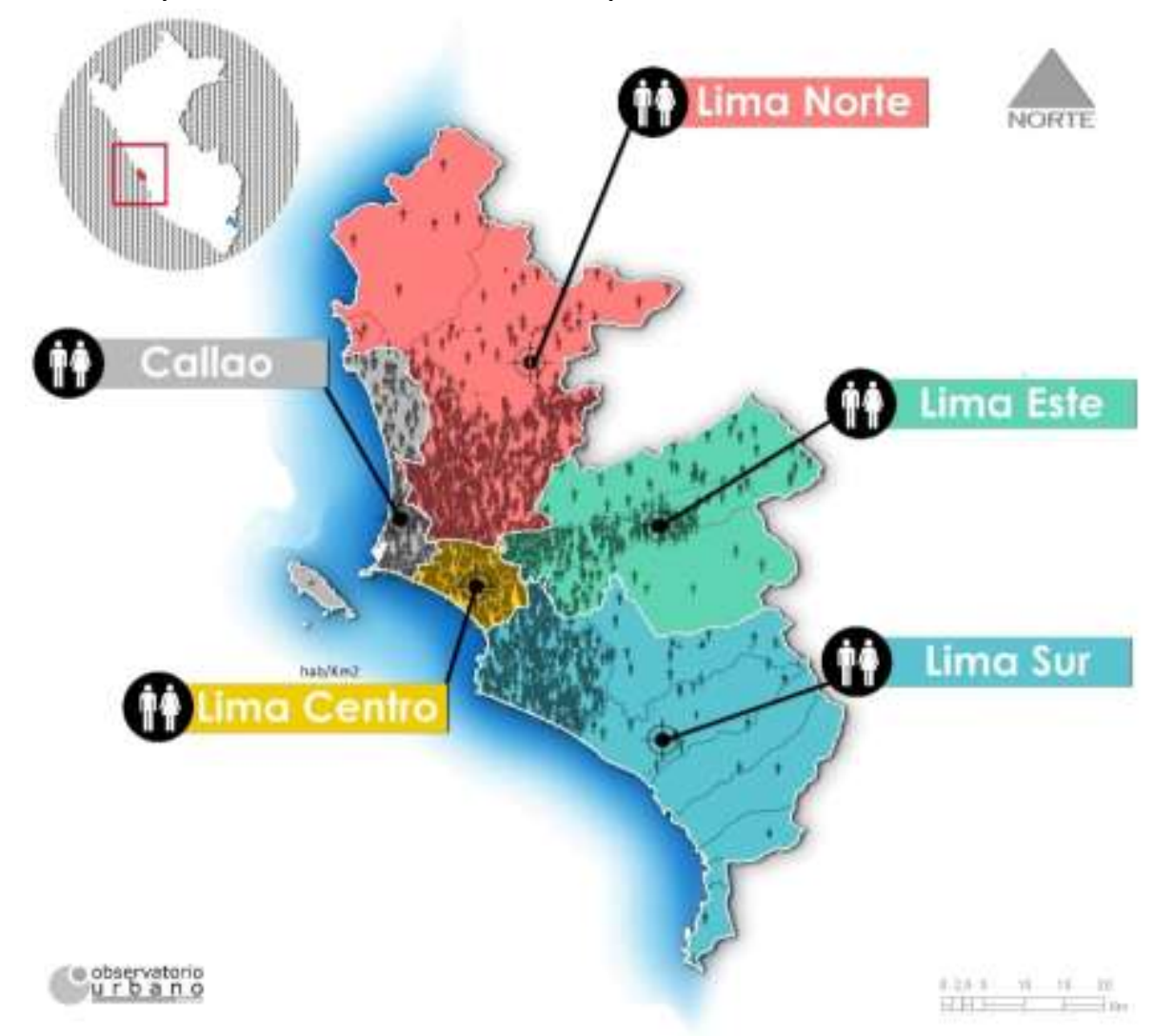

Fuente: adaptado de POBLACIÓN..., 2017.

La densidad poblacional dentro del territorio nacional peruano es de 24,2 hab./ km²; siendo mayor en los departamentos ubicados en la costa: Provincia Constitucional del Callao (6949 hab./km²), seguido del departamento de Lima $(282,4$ hab./km²) y en contraste encontramos los departamentos con menor densidad poblacional que se ubican en la selva 
como Madre de Dios $\left(1,6 \mathrm{hab} . / \mathrm{km}^{2}\right)$ y Loreto $\left(2,8 \mathrm{hab} . / \mathrm{km}^{2}\right)$ (INSTITUTO NACIONAL DE..., 2015).

\section{Uso y ocupación del suelo}

Las áreas agrícolas en el área metropolitana de Lima han disminuido notablemente en los últimos años; el mantenimiento de sus límites es difícil debido a la presión expansiva de ocupación del suelo para usos urbanos, a la precariedad legal de los usuarios y al sistema de comercialización.

Lima es una metrópoli con un patrón de ocupación territorial extensivo. La concentración de las actividades urbanas en determinadas áreas de la urbe y las relaciones que desarrollan los diversos grupos sociales van configurando el aspecto de dichos escenarios urbanos, definiendo la organización espacial del área metropolitana como monocéntrica y ramificada.

El centro de Lima es actualmente el eje de la actividad de los estratos bajos y medios de la población y su infraestructura urbana se mantiene, pero se utiliza de modo diferente. Este espacio urbano ha experimentado así un proceso de invasión-sucesión e invasiónsustitución que ha originado diversificación de actividades e intensificación de usos que lo han hecho disfuncional y de difícil control (PLAN..., 1992).

El crecimiento de las áreas periféricas (Lima Este, Lima Norte y Lima Sur), producido a partir de un modelo informal de ocupación del suelo es dinámico, en estas áreas han surgido ciertas actividades económicas propias (comercio informal, pequeña industria etc.) que tienden a retener parte de la fuerza laboral que no encuentra colocación en el sector formal de la economía. La marginalidad de las áreas periféricas ocupadas por estratos de menores ingresos se traduce en déficit de servicios básicos y de equipamiento urbano; dependencia económica, social y administrativa respecto al área central; desarticulación respecto de la trama urbana; insalubridad y alto riesgo; e inadecuada ocupación del espacio residencial.

\section{FACTORES QUE DESENCADENAN LA DINÁMICA DEMOGRÁFICA}

\section{Migración y aspectos históricos}

Uno de los fenómenos demográficos de importancia que caracteriza a la sociedad moderna es la gran movilidad espacial de la población. Sin embargo, no todo desplazamiento poblacional en el territorio puede ser considerado como migración. El Diccionario Demográfico Multilingüe (MACCIÓ, 1985) precisa para que tal hecho exista, se 
requiere el cruce de algún límite o frontera administrativa; así como el traslado de la residencia habitual (VILLA, 1991).

En todo el mundo el intenso crecimiento de la población urbana se presentó a partir de mediados del siglo pasado, cuando la economía mundial se mundializó y creció en tamaño generando el crecimiento a pasos agigantados de las ciudades alrededor del mundo. Así también, América Latina experimentó el proceso de crecimiento de las ciudades por el intenso proceso migratorio del campo a las ciudades,

[...] conllevando a la alta concentración de la población urbana. Este fenómeno migratorio se vio dinamizado por el ingreso a la transición demográfica debido a la disminución de la mortalidad y el incremento de la fecundidad, acelerándose el crecimiento poblacional y, como consecuencia, la crisis en el campo (INSTITUTO NACIONAL DE..., 2011, p. 31).

La historia de la migración en el Perú se relaciona con la crisis del campo, precipitando una gran oleada migratoria que en los siguientes 50 años cambió radicalmente la naturaleza de la sociedad peruana (MANRIQUE, 2004), siendo las ciudades los principales lugares de destino, sobre todo la ciudad de Lima. "Por vez primera, los peruanos del Ande, en su búsqueda del progreso, emigraron hacia la costa y empezaron a apropiarse de espacios físicos que antes les estaban vedados" (MÉNDEZ, 1997, p. 14).

La gran migración provinciana masiva a la costa y, principalmente, a Lima, se inició en la década de 1940, favorecida por la ampliación de la red vial y las transformaciones económicas que ensancharon al mercado interno. La expansión industrial internacional y la política modernizadora promovida por el gobierno militar de Odría permitieron la modernización del país y el incremento de las inversiones gubernamentales en obras públicas (MATOS, 1986). La mayor parte de estas inversiones se aplicó a planes de vivienda popular urbana, a la mejora de la infraestructura educativa y de la salud y al desarrollo de la red de carreteras.

En la década de 1950 se consolidaron los elementos centrales que caracterizan a la ciudad actual, la concentración de grandes contingentes de migrantes en Lima y demás ciudades; la expansión industrial y la ampliación de la presencia del Estado en todo el territorio. El auge de las exportaciones y el crecimiento de las importaciones multiplicaron la demanda de manufacturas y renovaron el impulso de la industrialización, creando nuevas fuentes de trabajo en la capital y principales ciudades costeñas, reforzando las tendencias migratorias. La miseria campesina y la difusión en el medio rural de información sobre los relativamente elevados niveles de vida de la capital y demás ciudades consolidaron una poderosa corriente migratoria del campo a la ciudad (MATOS, 1986). 
Gran parte de las ocupaciones de los espacios de Lima, llevadas a cabo por los migrantes, surgieron en terrenos desérticos que generalmente eran los cerros circundantes al centro de la ciudad, bastante lejos del casco urbano, formando así lo que hoy en día es Lima Este, Lima Norte y Lima Sur (figura 2). Estos espacios se desarrollaron en el límite de la zona urbana y la zona rural, siendo estos los espacios periurbanos de la ciudad, albergando dentro de ellos "[...] las barriadas de ayer, los pueblos jóvenes de un pasado reciente y los asentamientos humanos de hoy" (RAMIREZ, 2007, p. 322).

Figura 2 - Expansión urbana del área de Lima Metropolitana (1961 - 1981)

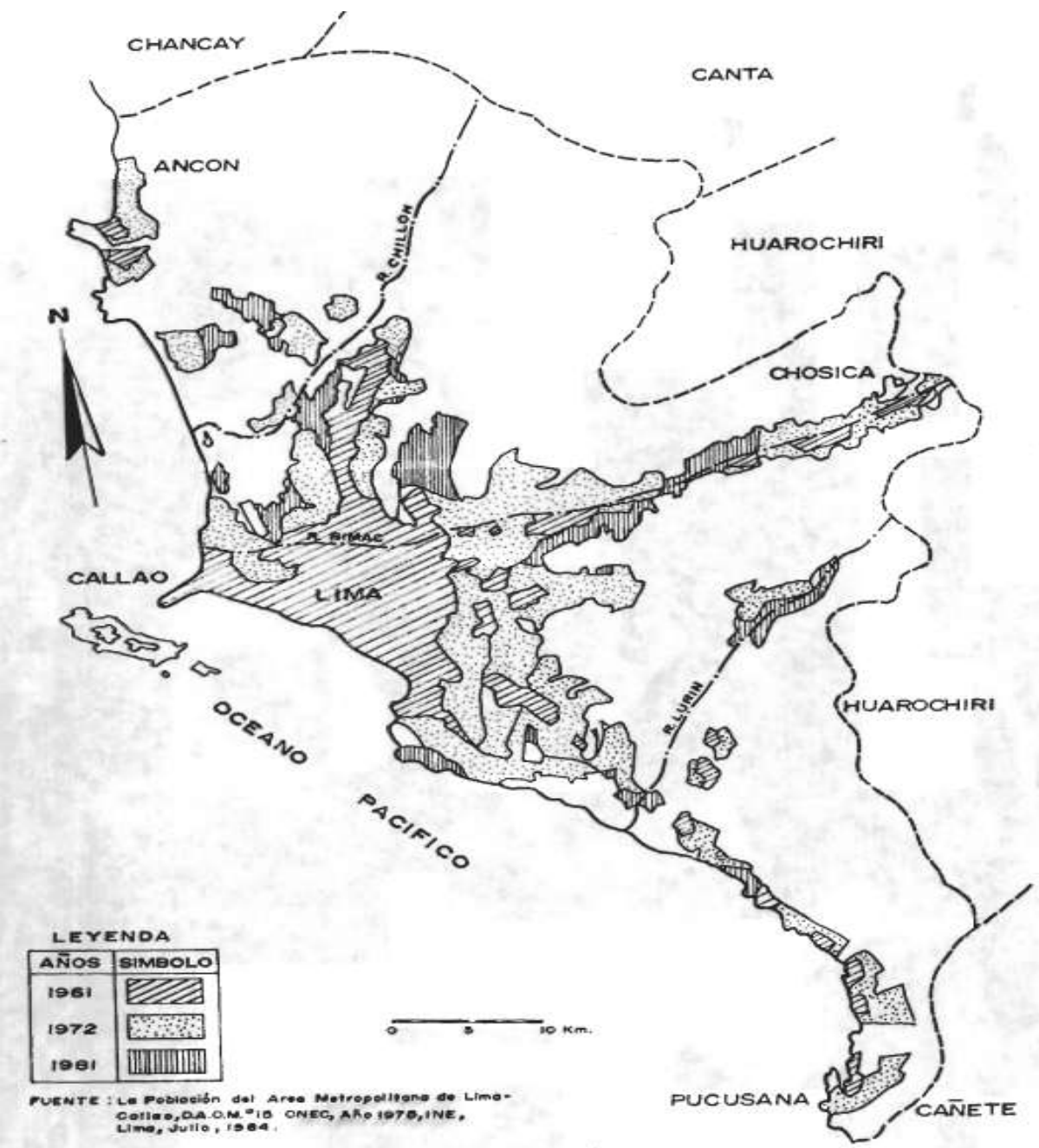

Fuente: PLAN..., 1992.

\section{Urbanización}

El proceso de urbanización en Lima Metropolitana se dio de forma acelerada a raíz de las grandes olas migratorias del campo a la ciudad, proceso que ocurría paralelamente alrededor del mundo desde mediados del siglo pasado. Se caracterizó desde sus inicios por ser desordenada, se invadieron terrenos que generalmente no estaban dentro del plan de 
urbanización del Estado por lo que cambiaron los usos del suelo ocupado, que en mayor parte fueron tierras agrícolas.

La migración interna del país provocó el crecimiento abrumador de la capital y la creciente concentración de la población en la periferia de la ciudad, dando paso a la creación de asentamientos humanos ubicados alrededor del centro de Lima, que se caracterizaban por su tamaño, roles y formas de organización. La aparición y creación de los asentamientos humanos, antes llamados barriadas y pueblos jóvenes en la periferia de la ciudad, está íntimamente vinculado a la urbanización acelerada de Lima Metropolitana, que fue una de las principales causas de atracción de las personas migrantes ante la escasez de viviendas y la pobreza que acechaba el interior del país.

El crecimiento de los asentamientos humanos en la zona periurbana de Lima ocurrió principalmente a partir de 1940 (tabla 1), evidenciándose en el aumento masivo de la población metropolitana que, de aproximadamente 645,000 habitantes en 1940, subió a 1 millón 845 mil 910 habitantes en 1961 y a 4 millones 608 mil 010 habitantes en 1981, tal como lo refleja los censos del Instituto Nacional de Estadística e Informática (1993).

Tabla 1 - Crecimiento poblacional nacional, regional y metropolitano (1940-1981)

\begin{tabular}{|c|c|c|c|c|c|c|c|c|}
\hline $\begin{array}{l}\text { REGIONES } \\
\text { NATURALES }\end{array}$ & $\begin{array}{r}1940 \\
\text { ABSOLUTA }\end{array}$ & $\%$ & $\begin{array}{r}1961 \\
\text { ABSOLUTA }\end{array}$ & $\%$ & $\begin{array}{r}1972 \\
\text { ABSOLUTA }\end{array}$ & $\%$ & $\begin{array}{r}1981 \\
\text { ABSOLUTA }\end{array}$ & $\%$ \\
\hline $\begin{array}{l}\text { República } \\
{ }^{*} \text { Región Costa } \\
\text {-Área Metropolitana }\end{array}$ & $\begin{array}{l}6,207,967 \\
1,759,573\end{array}$ & \begin{tabular}{c|}
100.0 \\
28.3
\end{tabular} & $\begin{array}{l}9,906,746 \\
3,859,443\end{array}$ & $\begin{array}{c}100.0 \\
39.0\end{array}$ & $\begin{array}{c}13,538,208 \\
6,242,993\end{array}$ & $\begin{array}{c}100.0 \\
46.1\end{array}$ & $\begin{array}{c}17,005,210 \\
8,477,687\end{array}$ & $\begin{array}{c}100.0 \\
50.0\end{array}$ \\
\hline $\begin{array}{l}\text { Lima Callao } \\
\text { - Resto de Costa } \\
{ }^{\star} \text { Región de la Sierra }\end{array}$ & $\begin{array}{c}645,172 \\
1,114,401 \\
4,033,942\end{array}$ & $\begin{array}{l}10.4 \\
17.9 \\
65.0\end{array}$ & $\begin{array}{l}1,845,910 \\
2,013,533 \\
5,182,093\end{array}$ & $\begin{array}{l}18.6 \\
20.4 \\
52.3\end{array}$ & $\begin{array}{l}3,302,523 \\
2,940,470 \\
5,953,293\end{array}$ & $\begin{array}{l}24.4 \\
21.7 \\
44.0\end{array}$ & $\begin{array}{l}4,608,010 \\
3,869,677 \\
6,716,012\end{array}$ & $\begin{array}{l}27.1 \\
22.9 \\
39.4\end{array}$ \\
\hline${ }^{\star}$ Región de la Selva & 414,452 & 6.7 & 865,21 & 8.7 & $1,341,922$ & 9.9 & $1,811,511$ & 10.6 \\
\hline
\end{tabular}

Fuente: Instituto Nacional de... (1993).

La migración del campo a la ciudad y la formación de asentamientos humanos circundantes a la ciudad contribuyeron en gran medida al proceso de urbanización como alivio de la escasez de vivienda. La gratuidad del terreno y la vivienda libre de alquiler en los asentamientos humanos permiten en cierta medida un beneficio para las familias de bajos ingresos económicos, especialmente en los periodos de desempleo y dolencias, ellos en su mayoría forman parte de la clase urbana pobre.

Los migrantes pobres se caracterizan en su mayoría por no poseer los conocimientos indispensables para desempeñarse en muchas de las ocupaciones urbanas, sin embargo, poseen una característica especial: la habilidad de construir sus propias viviendas en espacios que tienen rostro de urbano y alma de rural. Siendo ellos mismos los que diseñan sus viviendas en plena urbanización, tomando detalles de sus antiguas viviendas del campo, como dejar espacios libres dentro de la casa para la crianza de algunos animales 
domésticos y comestibles (cuyes, pollos, patos etc.), detalle que sirve como ayuda y alivio económico para las familias, pues crean sus propias fuentes de alimentación en medio de la crisis que permiten el desarrollo de una economía urbana de subsistencia (COLLIER, 1978).

Este proceso de urbanización acelerada que se da todavía hasta nuestros días y que se ve prioritariamente fomentada por la formación de los asentamientos humanos se escapa de las manos del Estado en su intento por controlarlos y regularlos, por lo que ofrece ciertos tipos de ayudas para mejorar las condiciones de vida de estos sectores populares urbanos, como por ejemplo: las facilidades para regularizar los procesos de formalización de viviendas, implementación de programas sociales frente a la pobreza, intentos por crear espacios de empleo etc. Todo ello que en su mayoría resulta ineficiente por su amplia demanda.

En ese sentido la urbanización como tal de los sectores populares urbanos ubicados en la periferia de la ciudad asume ventajas y muchas desventajas, por lo que el contraste de la gratuidad de la vivienda no es más grande que los problemas que suceden a partir de ello, éste rápido crecimiento periurbano requiere de grandes esfuerzos para mantener los niveles adecuados de una calidad de vida digna respecto a vivienda, empleo, alimentación y servicios de salud.

\section{Espacio periurbano}

Para referirnos y dar explicación a la conceptualización de la zona o espacio periurbano debemos primero estudiar los conceptos de urbano y rural. Comúnmente, cuando se habla de urbano y rural se suele relacionar ambos términos con actividades industriales y actividades agropecuarias respectivamente, donde uno muestra claramente un desarrollo definido a comparación del otro. Las diferentes concepciones entre lo rural y lo urbano siempre nos llevan a pensar en la línea que separa a ambos conceptos, una línea imaginaria que representa un trazo meramente territorial que generalmente está más vinculado a aspectos legales y jurídicos con fines de organización y tributación.

La división tradicional de los espacios urbano y rural es diseñada en función a los intereses y las perspectivas de desarrollo territorial de las instituciones estatales, resultando muchas veces el dominio de crecimiento de la expansión urbana sobre lo rural. Esa división que separa los espacios en una parte urbana y otra rural, por una línea definida como perímetro urbano, solo tiene validez en los puntos extremos, como criterio de división territorial (SPAROVEK, 2004). Pero ¿Qué es lo que realmente diferencia a una zona urbana de una zona rural? Generalmente la zona urbana está caracterizada por su alta densidad poblacional y por contar con servicios públicos y equipamientos urbanos como redes de infraestructura, transporte público, actividades comerciales e industriales; y la zona rural 
posee servicios públicos de manera limitada, se evidencia el predominio de las actividades agrícolas y áreas de preservación.

El espacio denominado zona periurbana, que como su nombre lo indica, se ubica en la periferia de la urbe llegando a ocupar espacios rurales por su extensión, que generalmente funciona como un espacio de interfaz entre lo urbano y lo rural, y se caracteriza por estar ubicado al margen de los bienes y servicios que se disponibilizan, encontrándose así los problemas de carácter socio-espacial del uso y ocupación del suelo. Este ámbito territorial puede ser conocido de distintas maneras y en la literatura se suele encontrar como: periurbano, exurbano, trazo urbano ("urban tract", rururbano, franja urbana, semi-urbano y aún, suburbano), aunque sus definiciones aún no están bien esclarecidas (IAQUINTA; DRESCHER, 2000). El autor de un estudio sobre periurbanización y espacios rurales en la periferia de las ciudades en México define periurbano como:

La extensión continúa de la ciudad y a la absorción paulatina de los espacios rurales que le rodean; se trata del ámbito de difusión urbano-rural e incluso rural, donde se desarrollan prácticas económicas y sociales ligadas a la dinámica de las ciudades. El elemento central en cuanto a la existencia del fenómeno lo constituyen las relaciones que se establecen por la cercanía y proximidad con el entorno urbano (ÁVILA, 2009, p. 98).

Podemos decir entonces que los espacios periurbanos son formas de organización espacial, extensiones urbanas hacia las zonas rurales, de carácter transicional donde se alternan los roles, poseyendo especial influencia del centro de la ciudad, por estar ubicados alrededor de la misma, dichos espacios por ser dispersos no poseen límites geográficos bien definidos. En estos espacios suele ocurrir la transformación de las áreas agrícolas absorbidas hacia patrones de usos discontinuos del suelo urbano-rural (AGUILAR, 2003).

\section{GEOGRAFÍA DE LA SALUD: TUBERCULOSIS}

Hablar de la geografía de la salud es importante para entender el proceso de la saludenfermedad, ello nos proporciona elementos para su comprensión y análisis teniendo como enfoque principal la relación de las enfermedades con el entorno, pasando las fronteras del enfoque biomédico, que explica la ocurrencia del proceso a partir de la biología humana, hasta llegar a un enfoque interdisciplinar, que explica la ocurrencia del proceso a partir del ambiente, estilos de vida y la distribución territorial.

La reproducción del espacio geográfico de habitación y las formas de vida benefician o perjudican la salud de las personas y comunidades, esto se ve determinado por la presencia y/o ausencia de saneamiento básico, contaminantes en el aire, condiciones del suelo, 
alimentación y recursos que facilitan el acceso a bienes y servicios (PERDOMO; CUARTAS, 2010).

La tuberculosis (TB) es la enfermedad que ha causado la mayor mortalidad entre los seres humanos a través de la historia (SELGELID, 2008). Estudios de paleopatología han demostrado la presencia del bacilo de Koch en momias de las culturas prehispánicas peruanas (GÓMEZ; SOUZA, 2003). Así también, desde el siglo XIX la TB fue identificada por Virchow y otros científicos de la época como una enfermedad social vinculada con la pobreza y con las malas condiciones de vida y de trabajo. La tuberculosis se concentra, principalmente, en las zonas en situación de pobreza y extrema pobreza. En estos espacios en particular, la baja calidad de vida y el hacinamiento tienen el poder de incrementar la diseminación de la bacteria y la exposición de las personas susceptibles a los casos infecciosos de la enfermedad. Sumado a ello, los más grandes problemas económicos surgen como resultado de los costos indirectos, tales como la pérdida del trabajo y productividad a causa de la enfermedad, lo cual convierte a una persona pobre en una persona enferma y mucho más pobre.

Lima y Callao concentran el $58 \%$ de pacientes de TB sensible (figura 3), el $82 \%$ de TB multidrogo resistente (MDR) y $93 \%$ de TB extensivamente resistente (XDR) del país (BONILLA, 2008). 
Figura 3 - Distritos con mayor concentración de casos de tuberculosis sensible - Lima Metropolitana, 2008

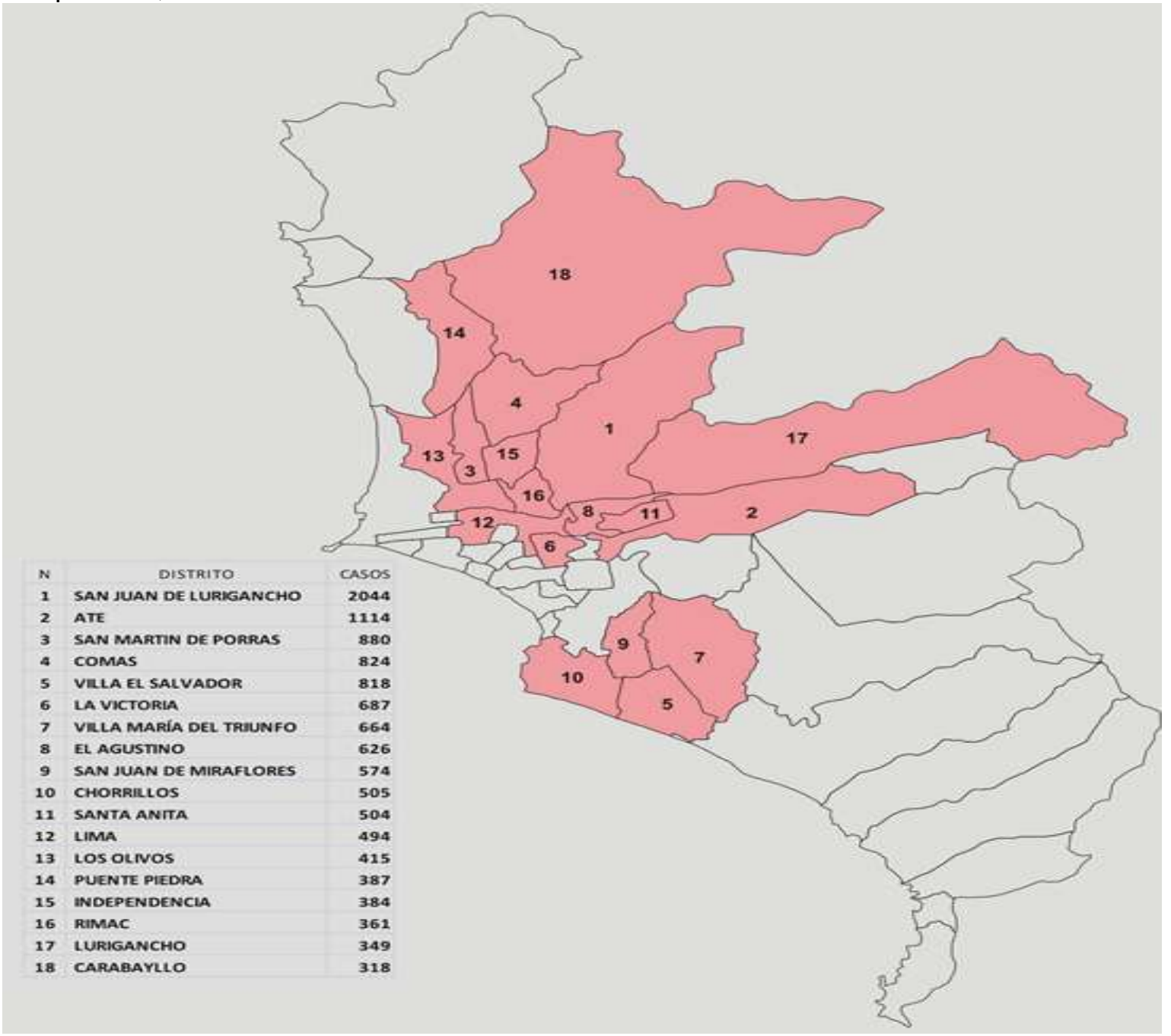

Fuente: Cabrera (2011).

\section{Ocupación del suelo y vivienda}

La ocupación de las áreas periurbanas de Lima se dio bajo un sistema de urbanización informal basado en invasiones colectivas, quienes carentes de viviendas ocuparon los valles y cerros, infringiendo muchas veces las normas estatales sin contemplación previa de procedimientos de planificación territorial ni de urbanización (saneamiento básico, manejo de desechos, ejecución de obras públicas etc.). La necesidad de contar con una vivienda hace que las personas, generalmente de bajas condiciones económicas, ocupen terrenos incluso en áreas de riesgo (PLAN..., 1992).

Las características de las viviendas de los asentamientos humanos ubicados en el espacio periurbano de Lima pueden agruparse en tres categorías, de acuerdo con la etapa de construcción que hayan alcanzado. La etapa de categoría inicial cuenta con muros y cobertura de material precario $41,8 \%$, entre ellos predomina la estera con $20 \%$, otros 
(cartón, latas, etc.) 13,4\%, madera 7,2\%, quincha y piedra/barro $0,7 \%$; la etapa de categoría medio cuenta con muros de ladrillo y concreto, sin techo de concreto $33,6 \%$ y la etapa de categoría básico cuenta con muros de ladrillo y techo de concreto $24,6 \%$ (INSTITUTO NACIONAL DE..., 1993).

En la actualidad, aproximadamente $40 \%$ de las viviendas en Lima tienen entre una y dos habitaciones y en $42 \%$ de ellas viven entre cuatro y seis personas, esto refleja las condiciones de hacinamiento en las que viven las personas (INSTITUTO NACIONAL DE..., 2008). La mayoría de las viviendas, de los asentamientos humanos de la zona periurbana de Lima, se encuentran caracterizadas en la etapa inicial y en condiciones de hacinamiento, ello constituye un factor de riesgo asociado a la tuberculosis ya que carecen de ventilación y entrada de la luz solar, facilitando la transmisión de la enfermedad.

\section{Condiciones de vida: pobreza e inequidad}

Las condiciones de vida de las personas enfermas con tuberculosis, que habitan los espacios periurbanos de Lima, son determinadas por un conjunto de problemas y necesidades insatisfechas que surgen a partir de las condiciones económicas y el posicionamiento territorial. Diversos estudios han relacionado la tuberculosis con la pobreza, siendo el principal determinante social de la enfermedad, esto se ve reflejado en la incidencia y prevalencia de la enfermedad en los países y ciudades con altos porcentajes de pobreza.

Perú presenta una tasa de pobreza de 23,9\% y Lima 14,8\% (INSTITUTO NACIONAL DE..., 2013), ubicando estos resultados mayormente en los distritos y asentamientos humanos de las zonas periurbanas (figura 4). Junto con la pobreza surgen factores que desencadenan la inequidad social y las personas afectadas con la enfermedad son aún más vulnerables a esta situación. La pobreza en las personas afectadas por tuberculosis agudiza su condición y las lleva a un estado más crítico pues ocasiona gasto económico, deterioro de la nutrición, desempleo, estigma social y pérdida de la autoestima. Todo ello genera que las personas afectadas tengan aún menos posibilidades de desarrollar sus potencialidades dentro de la sociedad. 
Figura 4 - Pobreza monetaria en Lima Metropolitana - 2007

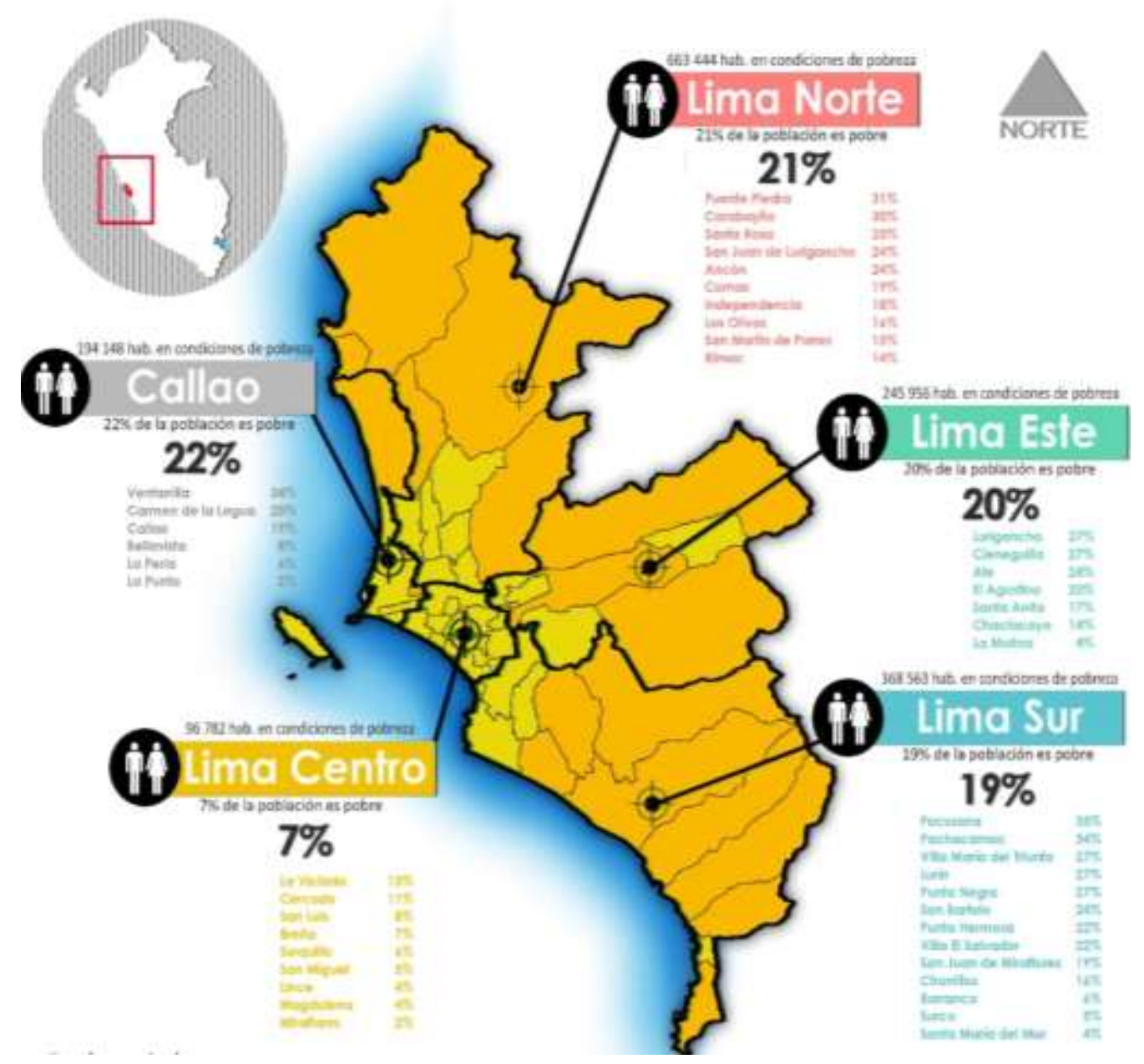

Fuente: Adaptado de POBREZA..., 2017.

\section{Necesidades básicas}

Las demandas de las necesidades básicas en una determinada población en interacción con la tuberculosis determinan la calidad de vida de las personas. Un informe del Instituto Nacional de Estadística e Informática (2014), respecto al acceso a los servicios básicos en los hogares de Lima Metropolitana del 2010 al 2013, registró que un 93\% de los hogares disponen de agua potable (figura 5), un 92\% disponen de desagüe (figura 6) y casi el $100 \%$ de los mismos acceden a la energía eléctrica (figura 7 ).

Así también, un 9,3\% de la población presenta al menos una necesidad básica insatisfecha, un $3,9 \%$ presenta viviendas con características físicas inadecuadas, los hogares presentan un $4,3 \%$ de hacinamiento y un $1,3 \%$ de las viviendas no cuentan con servicios higiénicos. Respecto al acceso a los servicios de salud, el 63,1\% de la población de Lima Metropolitana cuenta con algún tipo de seguro de salud.

Por otro lado, dentro de una sociedad salarial el empleo se convierte también en una necesidad básica que tienen los individuos sociales para garantizar sus condiciones de vida. 
En los afectados con tuberculosis surge la tendencia de pérdida del empleo, lo que acaba reconfigurando las relaciones familiares, por ejemplo, en el caso de los que tienen sobre sí la responsabilidad de mantenimiento económico de los miembros de su hogar pasa sobre otros (hijos, cónyuges o familiares cercanos) tal responsabilidad.

Figura 5 - Acceso a agua potable de los hogares de Lima Metropolitana, 2010 - 2013

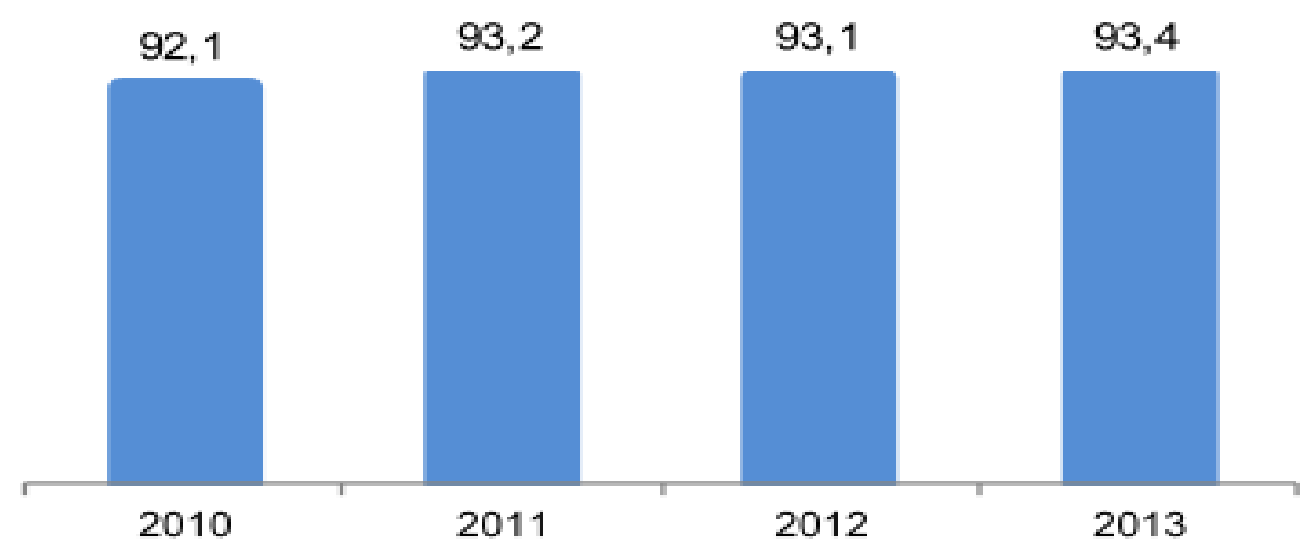

Fuente: Instituto Nacional de... (2014).

Figura 6 - Acceso a desagüe de los hogares de Lima Metropolitana, 2010 - 2013

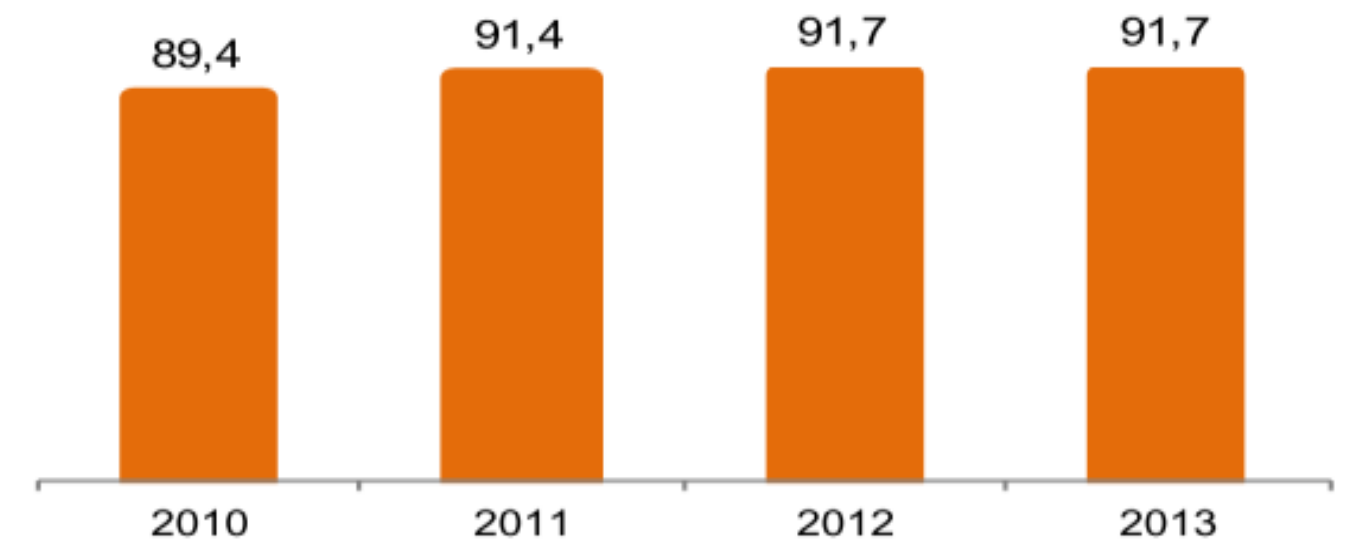

Fuente: Instituto Nacional de... (2014). 
Figura 7 - Acceso a energía eléctrica de los hogares de Lima Metropolitana, 2010 2013

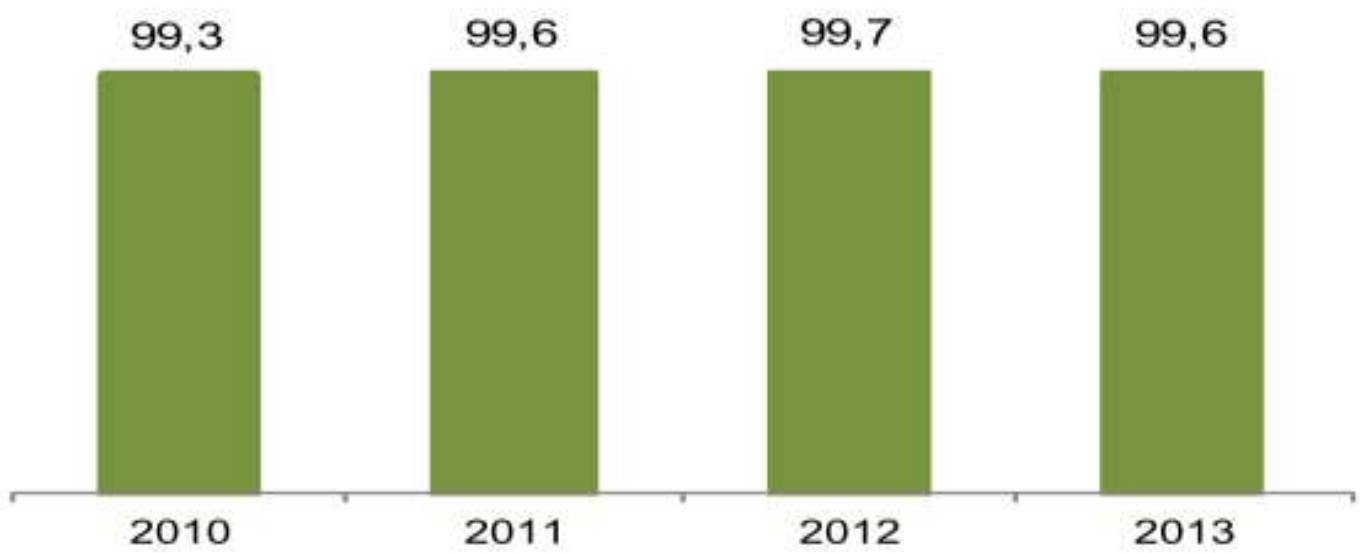

Fuente: Instituto Nacional de... (2014).

\section{Exclusión social}

La tuberculosis, la pobreza, la inequidad, la falta de acceso a los servicios básicos y otros determinantes sociales de la salud, en los espacios periurbanos de Lima, producen en los afectados niveles bajos de autoestima y pérdida de las capacidades de interacción en la sociedad generando exclusión y discriminación, esto ocurre visiblemente en nuestra sociedad que es discriminatoria y altamente jerarquizada (SULMONT, 2004).

Las personas afectadas por la tuberculosis son expuestas a tratos duales dentro de sus propias familias y comunidades (PERÚ, 2000). Reciben de un lado comprensión y soporte y por otro, señales de discriminación, por lo que van perdiendo prestigio y autoestima.

Todos estos signos deben ser tomados en cuenta por las instituciones que brindan el tratamiento al paciente para dar una atención más integral, un trato especial que brinde seguridad, bienestar y confort a los afectados por la enfermedad, de modo que puedan ir recuperando su autoestima e identidad, para que cuando llegue el día del término del tratamiento no tengan dificultades de insertarse nuevamente a la sociedad.

\section{CONSIDERACIONES FINALES}

Este trabajo tuvo como finalidad analizar los espacios periurbanos de Lima y su relación con la tuberculosis dentro de un abordaje social de la enfermedad. Por lo estudiado, podemos decir que la tuberculosis es una enfermedad social que tiene especial vínculo con la pobreza y con las deficientes condiciones de vida característicos de los espacios ocupados por la mayoría de migrantes, generando inequidad e injusticia social. Ello se traduce en la falta de acceso a los servicios básicos, a los servicios de salud, a la 
información y a un ambiente saludable, entre otros. La pobreza sumada a la enfermedad produce en las personas niveles bajos de autoestima y pérdida de las capacidades de interacción en la sociedad lo que genera, muchas veces, exclusión y discriminación.

En este espacio en particular se dan especiales procesos de transformación territorial y de condiciones de vida por ello será necesario orientar y promover el acondicionamiento territorial y el uso racional del suelo, preservando y mejorando las condiciones ambientales que lleven a mejorar las condiciones de salud de las personas afectadas con la enfermedad y de su entorno. El abordaje integral de la tuberculosis será fundamental para plantear intervenciones y soluciones eficaces, de modo que se implementen políticas públicas que atiendan y solucionen de manera eficiente la demanda de la enfermedad. La situación de salud de las personas se determina a partir de las condiciones de vida, del espacio ocupado, de los problemas de salud y de sus respuestas.

\section{REFERENCIAS}

AGUILAR, A. La megaurbanización en la Región Centro de México. Hacia un modelo de configuración territorial. En: . Urbanización, cambio tecnológico y costo social. El caso de la región centro de México. México: M. Á. Porrúa, 2003. p. 19-71.

ÁVILA, H. Periurbanización y espacios rurales en la periferia de las ciudades. Estudios Agrarios, Morelos, v. 15, n. 41, p. 93-123, mayo-ago. 2009.

BONILLA, C. Situación de la tuberculosis en el Perú. Acta Médica Peruana, Lima, v. 25, n. 3, p. 163-170, 2008.

CABRERA, T. Lima: sobredosis de TB. Lima, 2011. Disponible en:

<https://limamalalima.wordpress.com/2011/03/24/lima-sobredosis-de-tb/>. Acceso en: 15 dic. 2017.

COLLIER, D. Barriadas y élites: de Odría a Velasco. Lima: IEP, 1978.

GÓMEZ, J.; SOUZA, S. Prehistoric tuberculosis in America: adding comments to a literature review. Memórias do Instituto Oswaldo Cruz, Rio de Janeiro, v. 98, n. 1, p. 151-159, 2003. IAQUINTA, D.; DRESCHER, A. Defining Periurban: understanding Rural-Urban linkages and their connection to institucional contexts. Food and Agriculture Organization, Roma, v. 2000, n. 2, p. 8-26, 2000.

INSTITUTO NACIONAL DE ESTADÍSTICA E INFORMÁTICA - INEI. Anuario de Estadísticas Ambientales 2013. Lima, 2013. 1993.

Características sociodemográficas de los asentamientos humanos. Lima,

Censos nacionales de población y vivienda. Lima, 1993.

Migración Interna reciente y el Sistema de Ciudades, 2002 - 2007. Lima, 2011. 11 de Julio Día Mundial de la Población, Lima, 2015.

Perú: crecimiento y distribución de la población, 2007: censos nacionales 2007: XI de población y VI de vivienda. Lima, 2008.

Una mirada a Lima Metropolitana. Lima, 2014. 
MACCIÓ, G. A. Diccionario demográfico multilingüe: versión en español. 2 ed. Lieja: Ordina; 1985.

MANRIQUE, N. Enciclopedia temática del Perú: sociedad. Lima: El Comercio, 2004. 7 v. MATOS, J. Desborde popular y crisis del Estado. El nuevo rostro del Perú en la década de 1980. Lima: IEP; 1986.

Desborde popular y crisis del Estado. Veinte años después. Lima: Fondo Editorial del Congreso de la República, 2004.

MÉNDEZ, M. Migración, identidad y desarrollo: hacia una visión del país. Lima: IDL, 1997.

ORGANIZACIÓN MUNDIAL DE LA SALUD - OMS. Tuberculosis. 2016. Disponible en: <http://www.who.int/mediacentre/factsheets/fs104/es/>. Acceso en: 10 abr. 2017.

ORGANIZACIÓN PANAMERICANA DE SALUD - OPS. Tuberculosis en la Región de las Américas. Boletín, 2012.

PERDOMO, L.; CUARTAS, D. Encuentro de la geografía y la medicina, una visión entre el pasado y el presente como abordaje para el desarrollo de la distribución de las malformaciones congénitas mayores en Cali. Cali: Universidad del Valle, 2010.

Disponible en: <https://geografiadelasalud.blogia.com/2010/100301-encuentro-de-lageograf-a-y-la-medicina-una-visi-n-entre-el-pasado-y-el-present.php>. Acceso en: 8 mar. 2017.

PERÚ. Constitución Política del Perú de 1993. República del Perú, Lima, 1993. Disponible en: <http://www.wipo.int/wipolex/es/text.jsp?file_id=182579>. Acceso en: 8 mar. 2017.

PERÚ. Ministerio de Salud - MINSA. Estrategia Sanitaria Nacional de Prevención y Control de la Tuberculosis - ESNPCT. Situación de la tuberculosis en Lima, Perú. Lima, 2014.

PERÚ. Ministerio de Salud. Proyecto VIGIA. Vivir la tuberculosis. Estudio socioantropológico de la tuberculosis en dos zonas de alto riesgo en Lima: San Cosme y El Agustino. Lima: MINSA/USAID, 2000.

PLAN de desarrollo metropolitano de Lima-Callao 1990-2010 - PlanMet. Lima: SURP, 1992. POBLACIÓN en Lima Metropolitana. Disponible en:

<http://observatoriourbano.org.pe/project/poblacion-en-lima-metropolitana/>. Acceso en: 26 oct. 2017.

POBREZA monetaria en Lima Metropolitana. Disponible en:

<http://observatoriourbano.org.pe/project/pobreza-monetaria-en-lima-metropolitana/>.

Acceso en: 26 oct. 2017.

RAMIREZ, B. Desarrollo urbano y desigualdad en el área periurbana de Carabayllo. Investigaciones Sociales, Lima, v. 11, n. 18, p. 313-344. 2007.

SELGELID, M. Ethics, tuberculosis and globalization. Public Health Ethics, Oxford, v. 1, n. 1, p. 10-20, 2008.

SPAROVEK, G. et al. A linha imaginaria. In: SANTORO, P.; PINHEIRO, E. (Org.). O planejamento do município e o território rural. São Paulo: Instituto Pólis, 2004. p. 14-24.

SULMONT, D. Encuesta nacional sobre exclusión y discriminación social. Lima: DEMUS; 2004.

VILLA, M. Introducción al análisis de la migración. Santiago: CELADE, 1991.

Recebido: novembro de 2017.

Aceito: março de 2018. 